\title{
ВНУТРИКОСТНЫЙ ОСТЕОСИНТЕЗ КАК НОВАЯ ОПЦИЯ В ЛЕЧЕНИИ ПЕРЕЛОМОВ ПЯТОЧНОЙ КОСТИ
}

\author{
В.О. Каленский ${ }^{1}$, К.Ю. Кононова ${ }^{2}$, Д.А. Глухов ${ }^{2}$, П.А. Иванов ${ }^{1}$, \\ К.А. Бердюгин ${ }^{2}$, А.Н. Челноков ${ }^{2}$
}

${ }^{1}$ ГБУЗ «НИИ скорой помощи им. Н.В. Склифосовского Департамента здравоохранения г. Москвы», Большая Сухаревская площадь, д. 3, Москва, Россия,129010

${ }^{2}$ ФГБУ «Уральский научно-исследовательский институт травматологии и ортопедии им. В.Д. Чаклина» Минздрава России,

пер. Банковский, д. 7, г. Екатеринбург, Россия, 620014

\begin{abstract}
Резюме
Проведен ретроспективный анализ результатов лечения 36 пациентов (42 операции), которым был выполнен внутрикостный остеосинтез пяточной кости штифтом: на базе УНИИТО - 30 пациентов, на базе НИИСП 6 пациентов. Средний возраст пациентов составил 40,4 года. Билатеральные переломы диагностированы у 8 пациентов. У 4 пациентов имела место сочетанная травма. У всех пациентов переломы были закрытыми. В обоих лечебных учреждениях применялись сходные диагностический и лечебный алгоритмы. Предоперационное обследование включало рентгенографию и КТ. Оперативное вмешательство включало два этапа. Первым этапом выполнялась предварительная репозиция с помощью дистракционного аппарата и восстановление формы суставной поверхности через мини-доступы. Вторым этапом в пяточную кость вводили штифт и производили его блокирование. В послеоперационном периоде проводилась активная разработка движений в суставах стопы, пациенты обучались ходьбе при помощи костылей без нагрузки на поврежденную стопу. Сращение оценивали по контрольным рентгенограммам. Репозиция и элевация суставной площадки у всех пациентов, кроме одного, признана удовлетворительной. Средний срок наблюдения в серии УНИИТО составил 5,3 месяцев, в серии НИИСП - 9,8 месяцев. Ни у одного из пациентов в двух описанных сериях не наблюдалось гнойно-воспалительных осложнений и вторичного смещения отломков. В серии УНИИТО имел место один случай миграции винта. Консолидация наступила в срок от 2 до 3 месяцев после операции у всех пациентов.

Внутрикостный остеосинтез пяточной кости штифтом характеризуется сочетанием минимальной травматизации мягких тканей стопы, сохранением кровоснабжения латеральной кортикальной пластинки и стабильной фиксации, что значительно снижает риск инфекционных осложнений и расширяет показания к оперативному лечению. Применение данного метода возможно не только в условиях посттравматических местных нарушений трофики тканей, но и при наличии таких факторов риска, как сахарных диабет и курение. Для оценки функциональных исходов требуется продолжение исследования.
\end{abstract}

Ключевые слова: переломы пяточной кости, внутрикостный остеосинтез пяточной кости.

Переломы пяточной кости составляют $1,1-2,9 \%$ от всех повреждений костей скелета и $40-60 \%$ в структуре переломов костей стопы [7], около 75\% из них являются внутрисуставными [13]. По данным литературы, первичный выход на инвалидность при переломах пяточной кости составляет от 15 до 78\% [11]. Около 90\% больных данной категории - люди молодого и трудоспособного возраста от 18 до 55 лет [11]. Даже при использовании современных методов репозиции и фиксации неудовлетворительные исходы лечения тяжелых компрессионных переломов пяточной кости варьируют в диапазоне от $13 \%$ до 80,5\% [4, 8].
Определение оптимальной тактики лечения перелома пяточной кости нередко представляет для врача-травматолога сложную многофакторную задачу. Определение целесообразности, сроков и объема проведения оперативного вмешательства требует дифференцированного подхода. В настоящее время общепринятым считается проведение консервативного лечения и отказ от операции при переломах пяточной кости без смещения или с минимальным смещением отломков, а также, напротив, при многооскольчатых переломах, не подлежащих реконструкции (например, большинство переломов IV типа по классификации Sanders) [1, 3, 5-7].

미 Каленский В.О., Кононова К.Ю., Глухов Д.А., Иванов П.А., Бердюгин К.А., Челноков А.Н. Внутрикостный остеосинтез как новая опция в лечении переломов пяточной кости. Травматология и ортопедия России. 2015; (4):79-86.

$\bigotimes$ Каленский Всеволод Олегович. Большая Сухаревская площадь, д. 3, Москва, Россия,129010; e-mail: vsevolod.kalenskiy@gmail.com

1] Рукопись поступила: 08.06.2015; принята в печать: 29.09.2015 
В остальных случаях определение целесообразности операции проводится лечащим врачомтравматологом индивидуально с учетом рисков и технической возможности реконструкции.

Классический подход к остеосинтезу пяточной кости с использованием доступа по Essex - Lopresti, описанный в ряде авторитетных литературных источников, подразумевает большой L-образный разрез с выделением субпериостального лоскута и скелетированием латеральной стенки пяточной кости и, как следствие, ее деваскуляризации (рис. 1) [1, 5-7]. Остеосинтез пяточной кости в этих случаях проводится специальными пластинами. Достигается высокая стабильность фиксации и хорошее качество репозиции, так как операция проходит непосредственно под визуальным контролем хирурга, дополненным выполнением интраоперационной рентгенографии при помощи электронно-оптического преобразователя (ЭОП) (рис. 2).

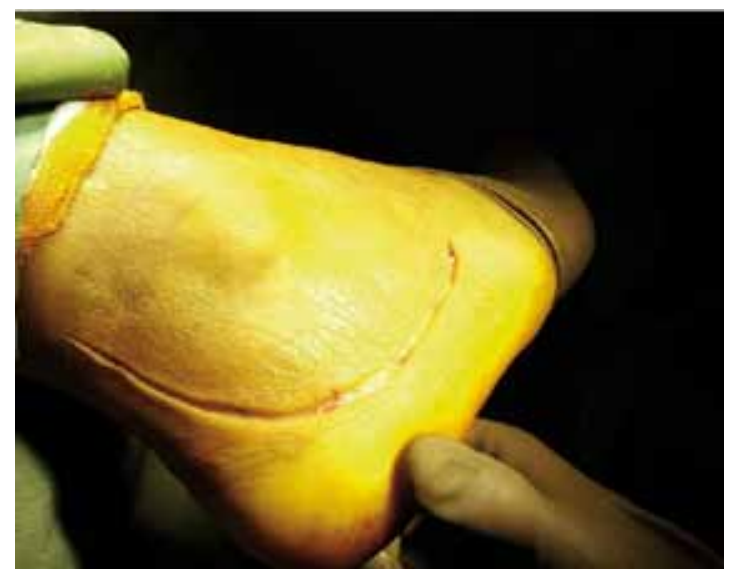

Рис. 1. Классический L-образный доступ Essex - Lopresti в модификации Tornetta

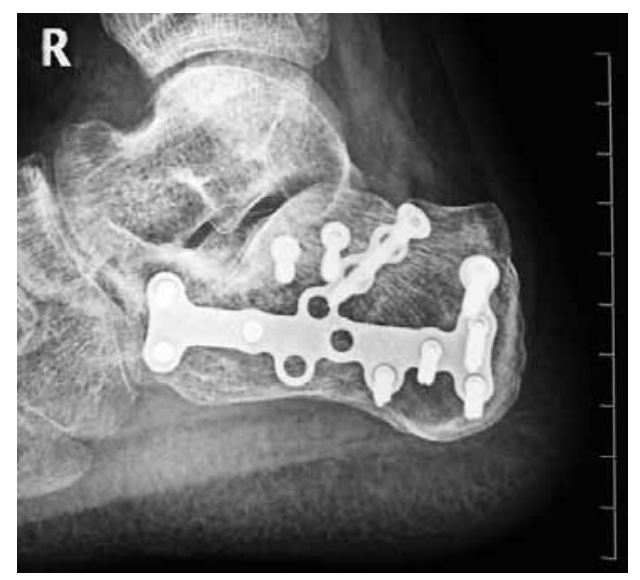

Рис. 2. Рентгенограмма пяточной кости после корректно выполненного остеосинтеза пластиной
Ряд факторов, как соматических (возраст, хроническая венозная недостаточность, анемия, курение и пр.), так и интраоперационных (высыхание лоскута во время операции, его сдавление инструментом, длительное время использования жгута и пр.), способны скомпрометировать кровообращение и привести к некрозу кожи и возникновению инфекционных осложнений в раннем послеоперацинном периоде (рис. 3) [1, 3, 5-7, 10, 14]. По данным ряда авторов, частота нарушений заживления послеоперационной раны в пяточной области может составлять до 25\% [5-8, 14].

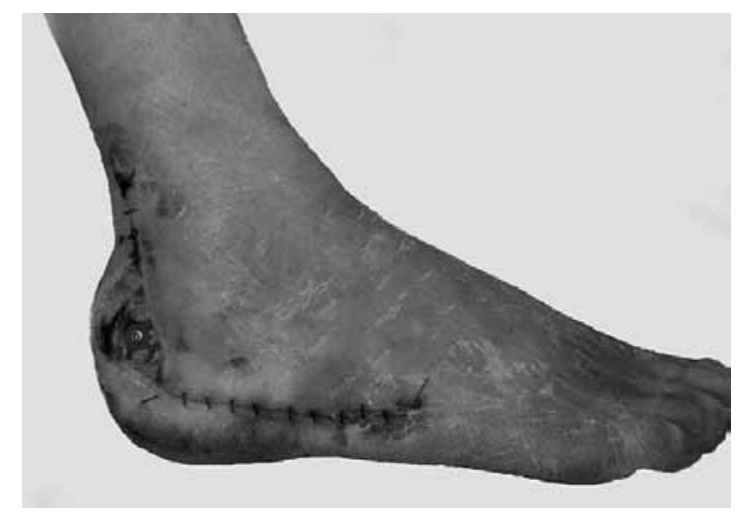

Рис. 3. Краевой некроз кожи, осложнившийся обнажением фиксатора и присоединением инфекции

Отдельную группу составляют пациенты, у которых переломы пяточных костей входят в состав сочетанной или множественной скелетной травмы. Сроки проведения операции на костях стопы у таких пациентов ввиду тяжести состояния смещаются на поздний период, когда мини-инвазивная хирургия становится технически неосуществимой. В то же время, в этой группе пациентов актуальна минимизация операционной травмы, так как риск осложнений ввиду посттравматической анемии и вторичного иммунодефицита значительно выше, чем у пациентов с изолированной травмой [12].

Существуют перкутанные техники репозиции, при использовании которых манипуляции осуществляются через проколы кожи без полноценного доступа. Винты и спицы, классически применяемые в этих случаях в качестве средств остеосинтеза, не всегда обеспечивают необходимую стабильность [5-7]. Применение аппарата Илизарова при переломах пяточной кости относится к мини-инвазивным манипуляциям, обеспечивает достаточную стабильность, но не решает проблему депрессии суставной площадки и нарушения ее формы. 
Перечисленные особенности и сложность соотношения рисков склоняют многих травматологов к выбору консервативного лечения, что может приводить к ухудшению функциональных исходов у пациентов, у которых возможна полноценная реконструкция пяточной кости. В последние годы несколько производителей травматологических имплантатов предложили варианты внурикостных конструкций для остеосинтеза пяточной кости, призванные решить вышеописанные проблемы [9].

Лечение по представленной методике проводилось одновременно в двух крупных травматологических стационарах: 2-м травматологическом отделении УНИИТО и в отделении сочетанной и множественной травмы НИИСП.

За 2013-2014 годы было проведено 42 операции остеосинтеза пяточных костей с применением пяточного штифта (35 в УНИИТО и 7 в НИИСП) у 36 пациентов (30 пациентов в УНИИТО и 6 пациентов в НИИСП). Критериями исключения являлись: недостаточное количество информации для анализа, потеря пациента из-под наблюдения, срок наблюдения менее 3 месяцев.

Соотношение мужчин и женщин в УНИИТО составило 27:3, в НИИСП - 4:2. Средний возраст пациентов в УНИИТО составил 40,4 года (от 22 до 62), пациентов НИИСП - 32,6 лет (от 20 до 40). В группе пациентов из НИИСП у 4 из 6 повреждение стопы входило в состав сочетанной травмы. Билатеральные переломы были диагностированы у 5 пациентов из УНИИТО и у 3 - из НИИСП. У всех пациентов в обеих сериях переломы пяточной кости носили закрытый характер. В серии УНИИТО в 4 случаях при КТ выявлен перелом IV типа по Sanders, в остальных 31 - III типа по Sanders. В серии НИИСП переломы пяточной кости без раскола суставной площадки (Sanders IIC) имели место в 3 случаях, переломы Sanders II А или В - в 3 случаях, перелом Sanders IIIAB - в 1 случае. Остеосинтез выполнялся в среднем на 21-е сутки в группе УНИИТО (от 5 до 45) и на 16-е сутки - в группе НИИСП (от 7 до 26).

Длительный предоперационный период в серии УНИИТО был обусловлен преимущественно поздним обращением, а в серии НИИСП - тяжелым состоянием пациентов, которое не позволяло провести оперативное вмешательство.

Все операции выполнялись без жгута, исключая 3 операции в НИИСП, при которых был выполнен доступ к подтаранному суставу. Среднее время операции составило 90 минут (от 60 до 190 минут) для серии УНИИТО и 133 минуты - для серии НИИСП (от 95 до 180 минут).
В качестве диагностических мероприятий на предоперационном этапе выполнялась рентгенография в боковой (рис. 4 a), аксиальной (по Harris) проекциях, а также проекциях по Broden. Выполнялась компьютерная томография (КТ) стопы с построением мультипланарных реконструкций и трехмерной модели в программе Multivox 5.0. Тип перелома по Sanders, степень внутрисуставного смещения оценивались по мультипланарным КТ-реконструкциям (рис. 4 б).
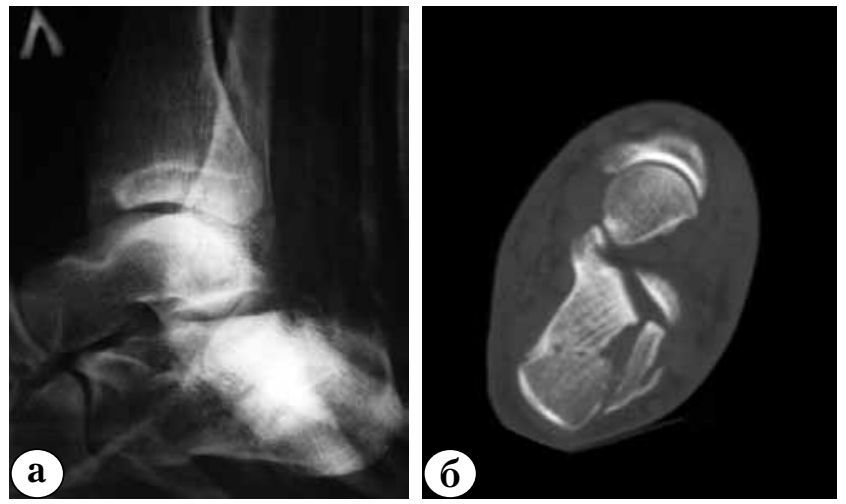

Рис. 4. Рентгенограмма в боковой проекции (a) и коронарная КТ-реконструкция (б), демонстрирующие перелом Sanders IIA у пациента 40 лет (серия НИИСП)

Техника операции. Операция включала два этапа. На первом этапе устанавливали дистракционный аппарат на двух спицах и двух опорах в виде полуколец, соединенных телескопическими тягами. Выполняли тракцию пяточного бугра в необходимом направлении. Особенность компоновки дистракционного аппарата обеспечивала достаточный обзор при рентгенографии (патент на полезную модель РФ 122011) (рис. 5). На втором этапе выполняли закрытую элевацию импрессированной суставной поверхности и окончательную репозицию пяточной кости. Через разрез длиной до 1 см по латеральному краю ахиллова сухожилия в месте его прикрепления к пяточной кости под соответствующий суставной фрагмент подводился элеватор, манипуляцией которым устраняли смещение (рис. 6). После элевации фрагмента выполняли провизорную фиксацию его спицей с последующим проведением субхондрального стягивающего винта (одного или двух). Пациентам из НИИСП в 3 случаях выполнена репозиция суставной поверхности задней фасетки через мини-доступ к подтаранному суставу. Также под контролем электронно-оптического преобразователя выполняли устранение углового (как правило, варусного) смещения бугра. 


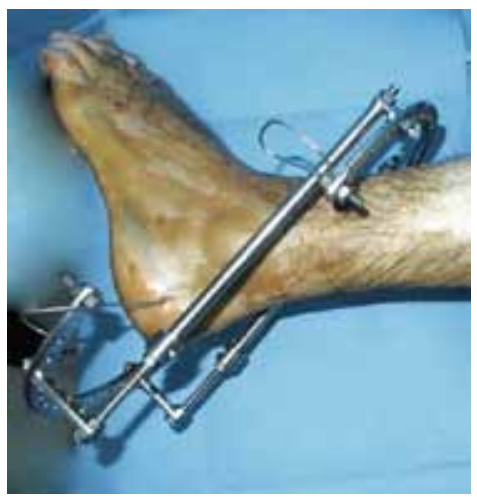

Рис. 5. Внешний вид дистракционного аппарата, применяемого на первом этапе
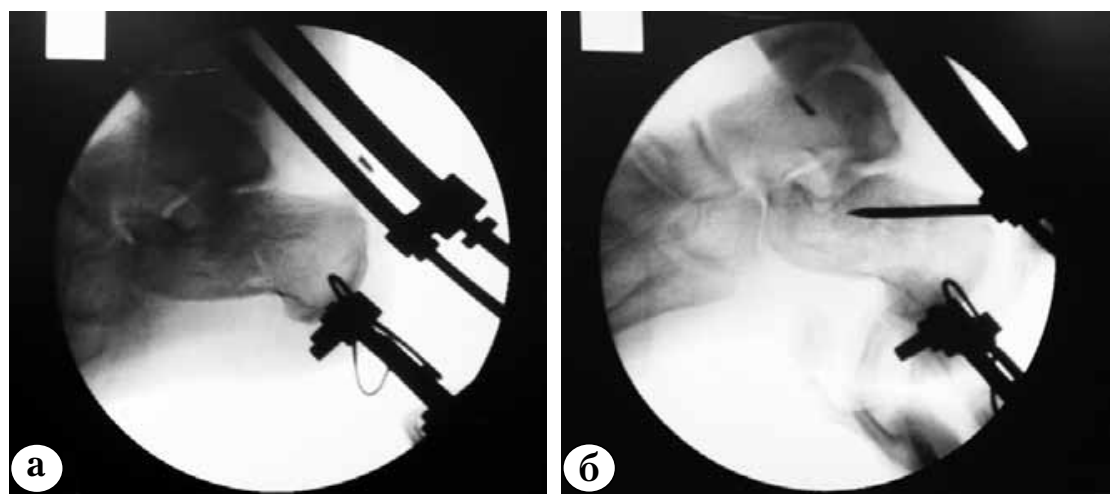

Рис. 6. Рентгенологическая картина после осуществления тракции в аппарате: импрессия суставной площадки (а) и элевация суставной площадки инструментом (б)
После завершения репозиции всех структур пяточной кости производилась установка штифта и его блокирование винтами (рис. 7). Данная методика репозиции и фиксации была впервые предложена коллективом авторов из УНИИТО [1], а позже внедрена в НИИСП. Во всех случаях был использован штифт C-nail (MEDIN, Чехия). Выбор конструкции обусловлен тем, что в настоящее время среди травматологических имплантатов, зарегистрированных в России, только этот тип внутрикостной конструкции позволяет обеспечить трехточечную фиксацию пяточной кости. В каждом из учреждений операции выполнялись одной хирургической бригадой, имеющей опыт как открытой, так и закрытой репозиции и фиксации пяточной кости.

Ни в одном наблюдении в обеих сериях не выполнялось замещение костных дефектов после элевации суставной поверхности, даже при значительных разрушениях пяточной

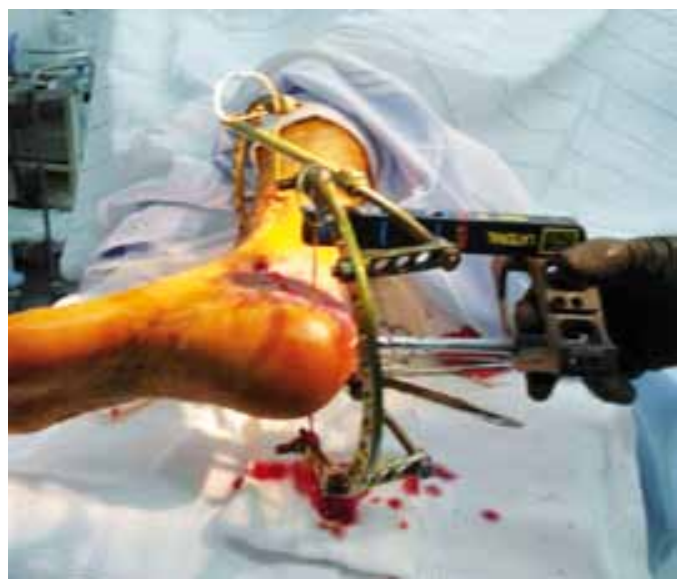

Рис. 7. Введение штифта после осуществления репозиции. Дистракционный аппарат не конфликтует с кондуктором штифта кости. Дренирование послеоперационной раны не проводилось.

Репозиция и элевация суставной площадки у пациентов в обеих сериях признана удовлетворительной (рис. 8). У одного пациента в серии НИИСП не достигнуто удовлетворительной элевации задней суставной площадки пяточной кости в связи с оскольчатым переломом переднего отдела пяточной кости и проседанием конструкции.
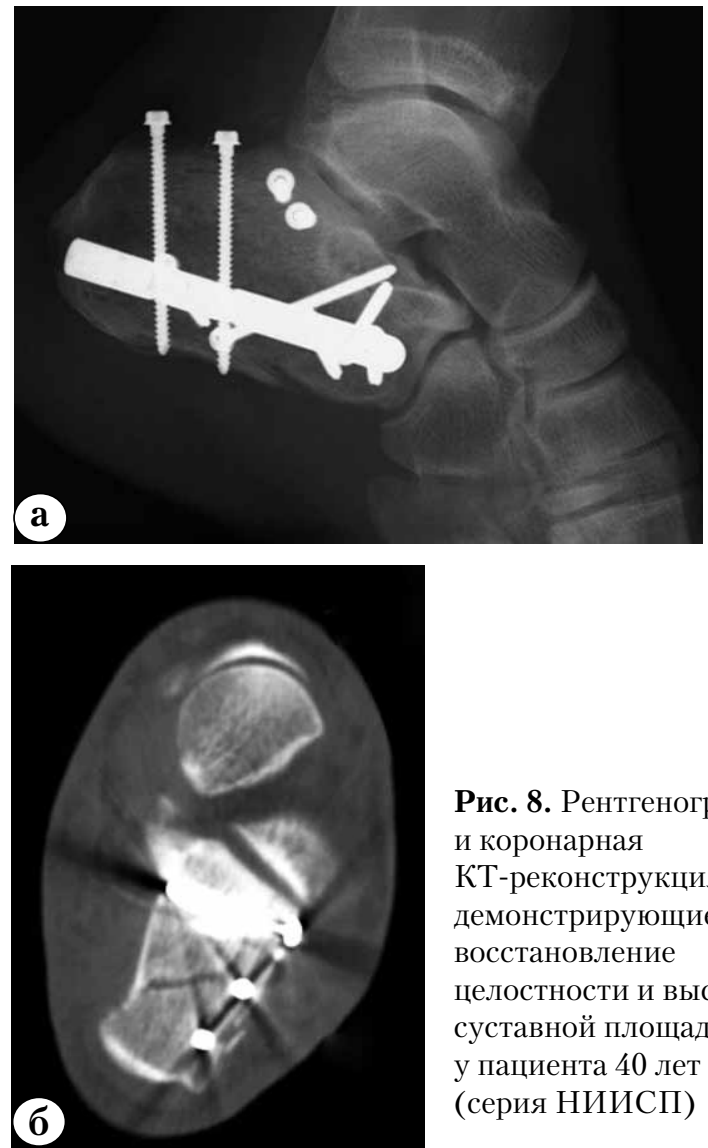

Рис. 8. Рентгенограмма (а) и коронарная КТ-реконструкция (б), демонстрирующие восстановление целостности и высоты суставной площадки у пациента 40 лет (серия НИИСП) 
В послеоперационном периоде внешняя иммобилизация не применялась. С 2-3-х суток пациентам рекомендована лечебная физкультура, направленная на восстановление объема движений в суставах стопы. В этот же срок пациенты обучались ходьбе при помощи костылей без нагрузки на поврежденную стопу (при отсутствии повреждений, не позволяющих ранний подъем и ходьбу). При билатеральных переломах пяточной кости, а также при повреждении контралатеральной нижней конечности пациенты начинали передвигаться в кресле-каталке. Наблюдение в послеоперационном периоде осуществлялось оперирующим хирургом. Контрольные рентгенограммы выполняли через 1, 2 и 3 месяца после операции. Консолидация перелома определялась по наличию костной мозоли, перекидывающейся через зону перелома. Сращение перелома определяли по данным рентгенографии. Полную нагрузку весом тела разрешали в сроки от 2,5 до 3 месяцев после операции на основании признаков консолидации на контрольных рентгенограммах (рис. 9).

Средний срок послеоперационного пребывания в стационаре составил 5,3 суток (от 4 до 10) в группе УНИИТО и 17 (от 5 до 45) -
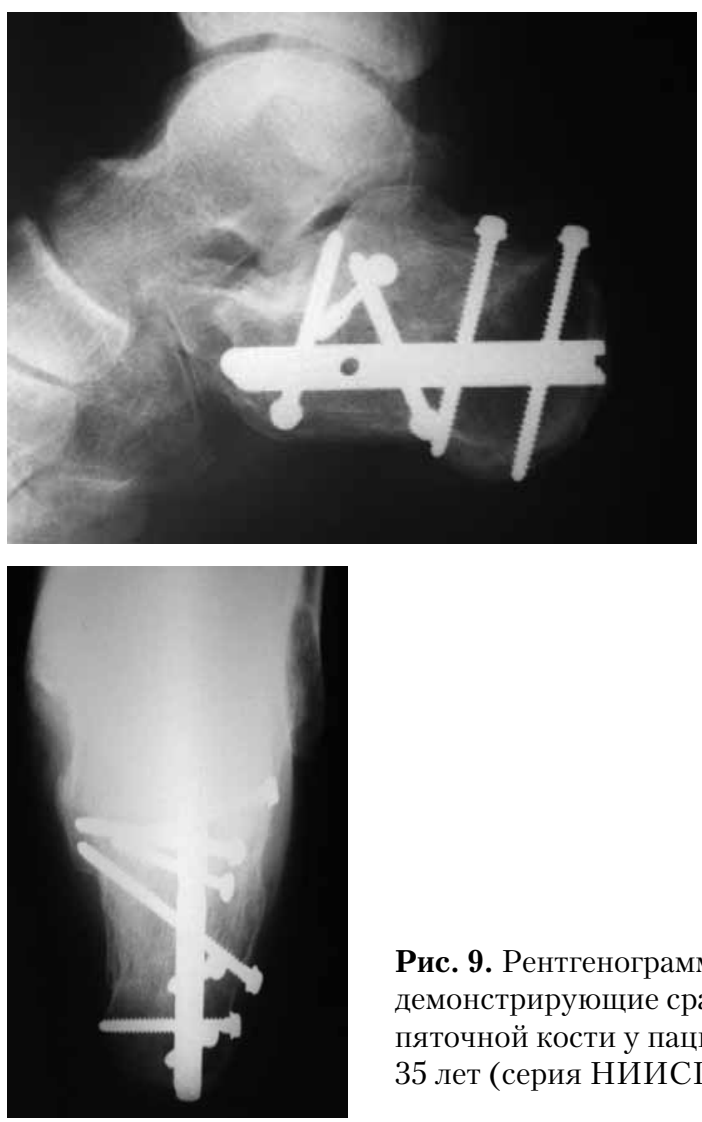

Рис. 9. Рентгенограммы, демонстрирующие сращение пяточной кости у пациента 35 лет (серия НИИСП) в группе НИИСП. Длительное послеоперационное пребывание в НИИСП было связано с наличием в серии пациентов с сочетанной травмой, которым требовались другие оперативные вмешательства. Средний срок наблюдения в группе УНИИТО составил 8,8 (от 1 до 20,5), в группе НИИСП - 9,8 месяцев (от 3,0 до 15,5). Ни у одного из пациентов в двух описанных сериях в раннем послеоперационном периоде не наблюдалось гнойно-воспалительных осложнений, краевых некрозов кожи и вторичного смещения отломков (рис. 10). В серии УНИИТО отмечен один случай миграции винта. Консолидация наступила в срок 2-3 месяца после операции у всех пациентов в обеих сериях.

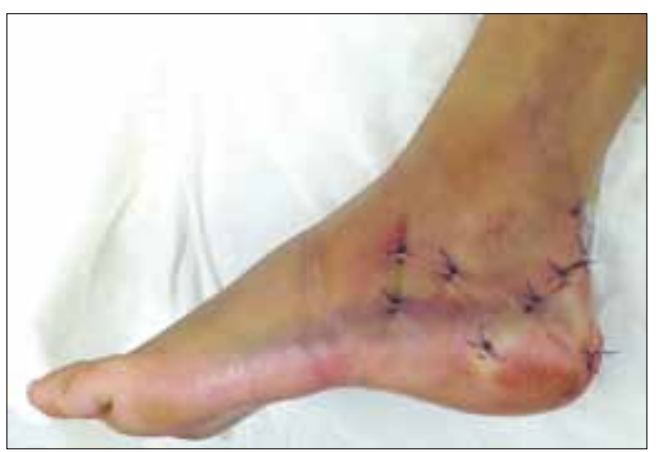

Рис. 10. Внешний вид стопы после перкутанной репозиции и установки штифта

В зависимости от клинической ситуации выбор тактики лечения пациентов с переломами пяточной кости варьирует от консервативного до полномасштабного травматичного хирургического вмешательства. Определение показаний к операции, сроков оперативного вмешательства, применяемых приемов репозиции и остеосинтеза основывается на множестве факторов, характеризующих как общее состояние пациента (тяжесть сопутствующих повреждений, состояние иммунной системы, наличие факторов риска), так и местный статус конечности (тип перелома, отрытый или закрытый характер перелома, локализация ран при открытых повреждениях, местные нарушения микроциркуляции и др.). R. Sanders и другие авторы, являющиеся апологетами накостного остеосинтеза пяточной кости, сообщают о $25 \%$ осложнений заживления раны у пациентов, имевших преимущественно закрытые изолированные переломы $[10,14]$. В случаях, когда вероятность возникновения осложнений велика, целесообразно применение мини-инвазивных хирургических техник, в число которых входит и остеосинтез пяточным штифтом. 
Нам не удалось найти публикаций, в которых бы сравнивались прочностные характеристики штифта, канюллированных винтов и спиц. Тем не менее, в отличие от винтов и спиц, исходя из своего строения, штифт обеспечивает стабильную трехточечную фиксацию, подобно современным пластинам с угловой стабильностью. M. Goldzak c соавторами при лабораторном механическом тестировании показали отсутствие отличий в стабильности фиксации между пяточным штифтом и пластиной с угловой стабильностью винтов [9].

В обеих сериях пациентов была достигнута удовлетворительная стабильность фиксации, а также отсутствовали типичные для данной анатомической области проблемы заживления послеоперационных ран.

\section{Выводы}

Внутрикостный остеосинтез пяточной кости штифтом представляется альтернативой классическому накостному остеосинтезу, а также существующим мини-инвазивным способам фиксации. Метод характеризуется сочетанием минимальной травматизации мягких тканей стопы, сохранением кровоснабжения латеральной кортикальной пластинки и стабильной фиксации.

Минимальная инвазивность репозиции и установки имплантата при достаточно прочной фиксации значительно снижает риск инфекционных осложнений (в наших наблюдениях их не отмечено), что расширяет показания к оперативному лечению и делает его возможным не только в условиях посттравматических местных нарушений трофики тканей, но и при наличии таких факторов риска, как сахарных диабет и курение, которые вынуждают отказываться от традиционного оперативного лечения с использованием пластин.

Представленные серии пациентов характеризуется коротким периодом наблюдения, не позволяющим произвести анализ функциональных исходов лечения, однако уже сейчас можно говорить о тенденции к малой частоте инфекционных осложнений и высокой стабиль- ности фиксации при использовании пяточного штифта. Целесообразно продолжение исследования данного метода.

Конфликт интересов: не заявлен.

\section{Литература}

1. Кононова К.Ю., Глухов Д.В., Бердюгин К.А., Челноков А.Н. Наш опыт лечения внутрисуставных переломов пяточной кости. Фундаментальные исследования. 2014; (10):294-297.

2. Коробушкин Г.В., Копенкин С.С., Скороглядов А.В., Плавунов Н.Ф. Выбор тактики лечения закрытых переломов пяточной кости. Вестник РГМУ. 2003; (5):55-59.

3. Корышков Н.А., Платонов С.М. Лечение повреждений пяточной кости: обзор литературы. Вестник травматологии и ортопедии им. Н.Н. Приорова. 2005; (1):90-92.

4. Asik M., Sen C. Surgical management of intraarticular fractures of the calcaneus. Arch Orthop Trauma Surg. 2002; 122(6):354-359.

5. Bucholz R.W., Heckman J.D., Court-Brown C.M., Tornetta P. Rockwood and Green's fractures in adults. 7th ed. Philadelphia: Lippincott Williams \& Wilkins; 2010.

6. Canale S.T., Boyd H.B., Beaty J.H. Campbell's operative orthopaedics 12th ed. Mosby, Elsevier. 2012.

7. Coughlin M.J., Mann R.A., Saltzman C. Surgery of the foot and ankle. 8th ed. Mosby, Elsevier. 2007.

8. Sabry F.F., Ebraheim N.A., Mehalik J.N., Rezcallah A.T. Internal architecture of the calcaneus: implication for calcaneus fractures. Foot Ankle Int. 2000; 21(2): 114-118.

9. Goldzak M., Simon P., Mittlmeier T., Chaussemier M., Chiergatti R. Primary stability of an intramedullary calcaneal nail and an angular stable calcaneal plate in a biomechanical testing model of intraarticular calcaneal fracture. Injury. 2014; 45, Suppl 1:49-53.

10. Harvey E.J., Grujic L., Early J.S., Benirschke S.K., Sangeorzan B.J. Morbidity associated with ORIF of intraarticular calcaneus fractures using a lateral approach. Foot Ankle Int. 2001; 22(11):868-873.

11. Hutchinson F., Huebener M.K. Treatment of the os calcis fracture by open reduction and internal fixation. Foot Ankle Int. 1994; 15(5):225-232.

12. Pape H.C., Sanders R., Borrelli Jr J. The poly-traumatized patient with fractures: a multi-disciplinary approach. Heidelberg: Springer; 2011.

13. Sanders R., Fortin P., DiPasquale T., Walling A. Operative treatment in 120 displaced intraarticular calcaneal fractures. Results using a prognostic computed tomography scan classification. Clin Orthop Relat Res. 1993; (290):87-95.

14. Swanson S.A., Clare M.P., Sanders R.W. Management of intra-articular fractures of the calcaneus. Foot Ankle Clin. 2008; 13(4):659-678.

\section{СВЕДЕНИЯ ОБ АВТОРАХ:}

Каленский Всеволод Олегович - младший научный сотрудник отделения сочетанной и множественной травмы ГБУЗ «НИИ скорой помощи им. Н.В. Склифосовского Департамента здравоохранения г. Москвы»

Кононова Ксения Юрьевна - аспирант клиники травматологии ФГБУ «Уральский научно-исследовательский институт травматологии и ортопедии им. В.Д. Чаклина» Минздрава России

Глухов Денис Валерьевич - канд. мед. наук старший научный сотрудник клиники травматологии, ФГБУ «Уральский научно-исследовательский институт травматологии и ортопедии им. В.Д. Чаклина» Минздрава России

Иванов Павел Анатольевич - д-р мед. наук заведующий научным отделением сочетанной и множественной травмы ГБУЗ «НИИ скорой помощи им. Н.В. Склифосовского Департамента здравоохранения г. Москвы» 
Бердюгин Кирилл Александрович - д-р мед. наук доцент, заместитель директора по научной работе ФГБУ «Уральский научно-исследовательский институт травматологии и ортопедии им. В.Д. Чаклина» Минздрава России

Челноков Александр Николаевич - канд. мед. наук руководитель клиники травматологии ФГБУ «Уральский научноисследовательский институт травматологии и ортопедии им. В.Д. Чаклина» Минздрава России

\title{
NAILING AS A NEW OPTION IN CALCANEAL FRACTURE TREATMENT
}

\author{
V.O. Kalensky ${ }^{1}$, K.Yu. Kononova ${ }^{2}$, D.A. Glukhov ${ }^{2}$, P.A. Ivanov ${ }^{1}$, \\ K.A. Berdyugin ${ }^{2}$, A.N. Chelnokov ${ }^{2}$ \\ ${ }^{1}$ Sklifosovsky Scientific Research Institute of Emergency Medicine, \\ Bol'shaya Sukharevskaya ploshchad', 3, Moscow, Russia,129010 \\ ${ }^{2}$ Chaklin Ural Scientific and Research Institute of Traumatology and Orthopaedics, \\ per. Bankozskiy, 7, Ekaterinburg, Russia, 620014
}

\begin{abstract}
Treatment of 36 patients in 2013-2014 with calcaneal fractures was retrospectively analyzed in two trauma centers (UNIITO and NIISP). Patients in both series were treated by similar algorithm. Diagnostic procedures included roentgenography and CT scan. Each operation had two stages. First one consisted of primary reduction by distraction device and precise reduction of posterior facet using miniapproaches. Second stage was nailing of calcaneus with interlocking. On 2-3 day after surgery active motions in ankle and subtalar joints were recommended. Weight bearing on damaged foot had been prohibited till bony union was achieved. Union was assessed by roentgenograms. 42 operations of calcaneal nailing were carried out in 36 patiens. Mean patient age in UNIITO series was 40,4 years, in NIISP series - 32,6 years. 5 patients in UNIITO series and 3 patients in NIISP series had bilateral fractures. 4 patients in NIISP series had polytrauma. There were no open fractures in both series. Reduction and elevation of posterior facet was acceptable in all patients in both series except 1 patient from NIISP. Mean follow up period was 5,3 month in UNIITO series and 9,8 month in NIISP series. No infection complications, skin necrosis or loss of fixation noted in both series. Screw migration met in 1 patient from UNIITO. Union achieved in all cases in $2-3$ month period.

There was a trend towards low infection rate and good mechanical stability. Nailing of calcaneus appears to be safe and effective alternative to classic methods. Futher investigation of this method is needed.
\end{abstract}

Key words: calcaneal fracture, osteosynthesis of calcaneus, nailing.

\section{Conflict of interest: none.}

\section{References}

1. Kononova KYu, Glukhov DV, Berdyugin KA, Chelnokov AN. [Our experience in intraarticular calcaneal fracture treatment]. Fundamental'nyye issledovaniya. [Fundamental Researches]. 2014; (10):294-297. [in Rus.]

2. Korobushkin GV, Kopenkin SS, Skoroglyadov AV, Plavunov NF. [Choice of tactics treatment of closed fractures of the calcaneus]. Vestnik RGMU [Bulletin of the Russian State Medical University]. 2003; (5):55-59 [in Rus.]

3. Koryshkov NA, Platonov SM. [Treatment of injuries of the calcaneus: a literature review]. Vestnik travmatologii i ortopedii im. N.N. Priorova [Priorov Bulletin of Traumatology and Orthopedics]. 2005; (1):90-92 [in Rus.]
4. Asik M, Sen C. Surgical management of intraarticular fractures of the calcaneus. Arch Orthop Trauma Surg. 2002; 122(6):354-359.

5. Bucholz RW, Heckman JD, Court-Brown CM, Tornetta P. Rockwood and Green's fractures in adults. 7th ed. Lippincott Williams \& Wilkins; 2010.

6. Canale ST, Boyd HB, Beaty JH. Campbell's Operative Orthopaedics 12th ed. Mosby, Elsevier; 2012.

7. Coughlin MJ, Mann RA, Saltzman C. Surgery of the Foot and Ankle. 8th ed. Mosby, Elsevier; 2007.

8. Sabry FF, Ebraheim NA, Mehalik JN, Rezcallah AT. Internal architecture of the calcaneus: implication for calcaneus fractures. Foot Ankle Int. 2000; 21(2):114-118.

9. Goldzak M, Simon P, Mittlmeier T, Chaussemier M, Chiergatti R. Primary stability of an intramedullary calcaneal nail andan angular stable calcaneal plate in a

[D] Cite as: Kalensky VO, Kononova KYu, Glukhov DA, Ivanov PA, Berdyugin KA, Chelnokov AN. [Nailing as a new option in calcaneal fracture treatment]. Travmatologiya i ortopediya Rossii. 2015; (4): 79-86. [in Russian]

$\triangle$ Kalensky Vsevolod O. Bol'shaya Sukharevskaya ploshchad', 3, Moscow, Russia,129010; e-mail: vsevolod.kalenskiy@gmail.com

i1 Received: 08.06.2015; Accepted for publication: 29.09.2015 
biomechanical testing model of intraarticular calcaneal fracture. Injury. 2014; 45, Suppl 1: 49-53.

10. Harvey EJ, Grujic L, Early JS, Benirschke SK, Sangeorzan BJ. Morbidity associated with ORIF of intraarticular calcaneus fractures using a lateral approach. Foot Ankle Int. 2001; 22(11):868-873.

11. Hutchinson F, Huebener MK. Treatment of the os calcis fracture by open reduction and internal fixation. Foot Ankle Int. 1994; 15(5):225-232.
12. Pape HC, Sanders R, Borrelli Jr J, eds. The Polytraumatized Patient with Fractures. Springer; 2011.

13. Sanders R, Fortin P, DiPasquale T, Walling A. Operative treatment in 120 displaced intraarticular calcaneal fractures. Results using a prognostic computed tomography scan classification. Clin Orthop Relat Res. 1993; (290):87-95.

14. Swanson SA, Clare MP, Sanders RW. Management of intra-articular fractures of the calcaneus. Foot Ankle Clin. 2008; 13(4):659-678.

\section{INFORMATION ABOUT AUTHORS:} Care

Kalensky Vsevolod O. - researcher of polytrauma department, Sklifosovsky Scientific and Research Institute of Emergency

Kononova Kseniya Yu. - postgraduate, trauma department, Chaklin Ural Scientific and Research Institute of Traumatology and Orthopaedics

Glukhov Denis V. - senior researcher of trauma department, Chaklin Ural Scientific and Research Institute of Traumatology and Orthopaedics

Ivanov Pavel A. - head of polytrauma department, Sklifosovsky Scientific and Research Institute of Emergency Care

Berdyugin Kirill A. - deputy director of scientific work, Chaklin Ural Scientific and Research Institute of Traumatology and Orthopaedics

Chelnokov Aleksandr N. - head of trauma department, Chaklin Ural Scientific and Research Institute of Traumatology and Orthopaedics 